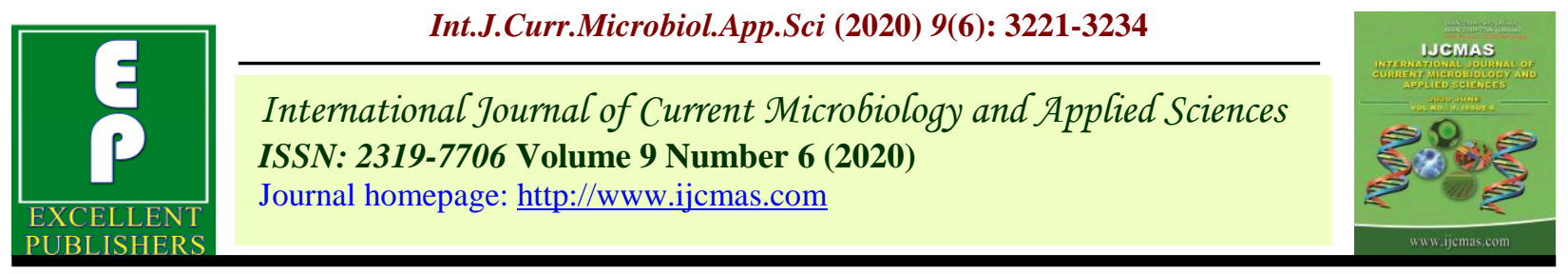

\title{
Extent of Media Use by Women Members of PRIs for Seeking Information on Rural Development
}

\author{
S. Kanase Pallavi ${ }^{1}$, R.B. Kalamkar ${ }^{2 *}$, S.P. Gaikwad ${ }^{2}$ and K.V. Chorge ${ }^{3}$ \\ ${ }^{1}$ Extension Education, RCSMC College of ABM, Sangli, India \\ ${ }^{2}$ Extension Education, College of Agriculture, Baramati, India \\ ${ }^{3}$ Department of Extension Education, College of Agriculture, Dr Balasaheb Sawant Konkan \\ Krishi Vidyapeeth, Dapoli, 415712, India
}

*Corresponding author

\begin{tabular}{|l|}
\hline K e y w o r d s \\
Panchayat Raj \\
Institutions (PRIs), \\
Extent of \\
media use \\
\hline Article Info \\
\hline Accepted: \\
21 May 2020 \\
Available Online: \\
10 June 2020 \\
\hline
\end{tabular}

\section{A B S T R A C T}

The study was conducted in Ratnagiri district. In all, 112 PRI woman members were selected from four Panchayat Samities, five Grampanchayats from each selected Panchayat Samiti and Ratnagiri Zilha Parishad on the basis of maximum number of woman members. They were interviewed with the help of a specially designed interview schedule. An interview schedule was prepared in Marathi to collect the information in line with the objective of the study. Collected data were processed and tabulated by using simple frequency, and the parameters like percentage, mean and standard deviation, as well as, the correlation and regression coefficient were used. The PRI woman members used more personal and mass media for seeking information. The respondent's media utilization was mostly for agriculture, education, health, women and child welfare and other developmental activities.

\section{Introduction}

After seventy two years of independence, rural women are still ignorant about many activities of their day to day life. As per the $73^{\text {rd }}$ constitutional amendment, 33 per cent seats are reserved for women in Panchayat Raj Institution. The Ashok Mehta Committee Report (1978) stressed the importance of role of women in decision making in development.
In fact, for the first time, the Committee with all the women member of the zillha parishad represented on it, so as to enable the women to make the decisions themselves on priorities and choices in welfare and development programmes meant for women and children. Many developmental programmes are being launched by government and non-government organization for rural people. Hence, there is an urgent need to diffuse the useful technical 
know-how among PRIs woman members to improve socio-economic status and increase their knowledge level.

Members elected to the various PRIs are important, not only because they are politically influential, but also because they are direct carriers of knowledge of various agricultural and rural development programmes and modern agricultural techniques to rural community. Any community has a tendency to follow their leaders. At grass root level, the three tier system of Panchayat Raj Institution is the foremost system of our republic government from grampanchayat at village level, panchayat samiti at taluka level and zilla parishad at district level. The persons elected as members of these institutions play a very important role in the process of rural development.

\section{Statement of the problem}

The Panchayat Raj has come into existence with the sole aim of decentralization of governmental power for the welfare of the rural people. After independence, the Panchayat Raj has become a back-bone of Indian democracy. Indian women were often 'physically visible' but conceptually 'invisible' and were remained marginalized. But now a days, situation is changing; they are coming in each sector with men and thus in administration also.

Union Cabinet of Government of India on 27 August 2009, approved 50 per cent reservation for women in PRIs. The Indian states those have already implemented 50 per cent reservation are Madhya Pradesh, Bihar, Uttarakhand and Himachal Pradesh. Also on 25 of November 2011, the states of Andhra Pradesh, Chhatisgarh, Jharkhand, Kerala, Maharashtra, Orissa, Rajasthan and Tripura are also having 50 per cent reservation for women in Panchayat Raj. The women
Panchayat Chairpersons in many places are doing well, there is only need to disseminate information regarding various aspects of rural development. This can be only possible with help of communication media.

Therefore, the present study was conducted with objectives To understand extent of media use by woman members of PRIs for seeking information on rural development

\section{Limitations of the study}

Due to limitation of time and resources, the study was restricted to limited aspects. Also, the data were collected from one Zilla Parishad, four Panchayat Samities and five Grampanchayats from each selected tahsils in Ratnagiri district. Hence, findings cannot be generalized beyond the limits of the area of the study. However, the findings may become applicable in the areas where similar conditions exist.

\section{Area of study}

The study was conducted in the Ratnagiri district of Konkan region having geographical area 8,208 sq.km. There are nine tahsils, in the district. The study was carried out in four tahsils namely, Ratnagiri, Chiplun, Khed and Dapoli.

\section{Tools and techniques of data collection}

An interview schedule was prepared in Marathi to collect the information in line with the objectives of the study.

\section{Statistical analysis}

The data were processed and tabulated by using simple frequency, and the parameters like percentage, mean and standard deviation, as well as, the correlation and regression coefficient were used. 


\section{Results and Discussion}

\section{Overall extent of media use}

The data regarding, extent of media use by the respondents are given in Table 1.

It is observed from Table 1 that majority of the respondents (70.54 per cent) had 'medium' extent of media use, while remaining 15.18 per cent and 14.28 per cent respondents had 'low' and 'high' extent of media use. The average media use score of the respondents was 31.65 indicating 'medium' use.

The investigation suggests that the woman members of PRIs should be trained and motivated to make maximum use of available media.

The findings are similar with the findings of Mali (2004).

\section{Type and frequency of media use}

The data regarding type and frequency of media use by the respondents are as given in Table 2.

It is observed from Table 2 that among localite personal contact media, large majority (92.86 per cent) of the respondents 'always' used family member for seeking information on rural development, followed by other important sources as Grampanchayat members (56.25 per cent), local leader (44.64 per cent), neighbor (32.14 per cent), Panchayat Samiti members (24.10 per cent). Whereas majority (80.36 per cent) of the respondents 'sometimes' used 'relative' for seeking information on rural development followed by other important sources as progressive farmers (77.68 per cent), friends( 68.75 per cent), neighbour (48.22 per cent), local leaders (44.64 per cent) and
Grampanchayat members(36.60 per cent). Majority of the respondents (78.57 per cent) 'never' contacted Zillha Parishad Chairperson for information seeking, followed by other important sources as Zillha Parishad members (73.21 per cent), Panchayat Samiti President (67.86 per cent) and Panchayat Samiti members( 65.18 per cent). It seems that woman members had comparatively less contact to non-officials of PRIs.

Regarding personal contact with Department of Agriculture personnel, it was observed that very few (10.71per cent) respondents 'always' used Taluka Agriculture Officer for seeking information, followed by Circle Agriculture officer (8.93 per cent), District Superintend Agriculture Officer (3.58 per cent), Agriculture Assistant (2.68 per cent). Respondents 'sometimes' used the media namely, Circle Agriculture Officer (81.25 per cent), followed by Agriculture Assistant (58.93 per cent) and Taluka Agriculture Officer (46.43 per cent). Majority of the respondents 'never' used the media such as Subdivisional Agriculture Officers (90.19 per cent), followed by Agriculture Supervisor (86.61 per cent) and District S. A.O. (84.82 per cent).

Among Panchayat Raj staff/ officers, majority (66.07 per cent) of the respondents 'always' had contacted with 'Gramsevak' for information seeking, followed by Agriculture Officers (16.97 per cent), Block Development Officer (18.75 per cent) and Agricultural Extension Officer (12.50 per cent). Majority of them 'sometimes' used these media namely Agriculture Officers (66.96 per cent), Block Development Officer (60.72 per cent). Majority of the respondents (81.25 per cent) 'never' contacted Project Officer for information seeking.

Respondent's contact with personnel from agriculture universities for seeking 
information was found less. As per the table, only 1.78 per cent respondents had 'always' contacted the SMS (subject matter specialist) and scientists, followed by Agriculture Assistant (0.89 per cent).

The study revealed that there was 'negligible' or 'less' contact with Bank Officers for seeking information by PRI woman members. Only 4.46 per cent respondents had 'sometimes' contacted the Branch Manager of Bank, while none had contacted the Agriculture Officer of bank for information seeking purpose.

In case of mass media, majority ( 74.10 per cent) of the respondents 'always' used newspaper, followed by television (59.82 per cent), newsletters (36.61 per cent) and Radio (25.00 per cent). Majority of the respondents 'sometimes' used for seeking information followed by agriculture magazines $(74.10$ per cent), newsletters (58.04 per cent), radio (40.18 per cent), agriculture diary(39.28 per cent) and television(36.61 per cent). Majority of the respondents 'never' used mass media are as journals (88.39 per cent), internet (87.50 per cent), computer (79.47 per cent), books on schemes (72.33 per cent). It means the woman members were using the media readily available to them.

Among the extension teaching methods, majority (67.86 per cent) of the respondents 'always' used circulars for information seeking, followed by group discussion (60.71 per cent). Majority (95.54 per cent) of the respondents 'sometimes' used campaign, followed by exhibition and posters (91.08 per cent each), rally (84.82 per cent), training (79.46 per cent), charts (60.72 per cent) and visits (45.54 per cent). Majority (95.54 per cent) of the respondents 'never' used either 'trials' or 'workshops', followed by seminar (94.84 per cent), tape recorder (94.64 per cent) and graphs (93.75 per cent).
It can be said that majority of the respondents 'always' consulted the 'family members. It may be due to the reason that our Indian women are less cosmopolite. They spend their maximum time with family members, relatives, neighbours who are localite in nature. Also, majority of the respondents 'sometimes' contacted the progressive farmers for seeking information as majority of families have 'farming' is major occupation, also the housewives help in farming. There is considerable contact with Grampanchayat members for seeking information, as they themselves are members of PRIs.

Inferences can be drawn from these findings that less respondents contacted to personnel from Department of Agriculture. Majority of the respondents 'sometimes' contacted them for seeking agricultural information various schemes, arranging visits/exhibitions. They contacted the Taluka Agriculture Officer and others.

From the Panchayat Raj staff, majority 'always' consulted to Gramsevak for seeking information regarding rural development as he is the Secretary of 'Grampanchayat'. Also he is the most accessible source of information about all government schemes and programmes at grassroot level and their implementation is done through him.

Also, Agriculture Officers, Block Development Officer, Agricultural Extension Officer, were contacted 'sometimes' by considerable respondents, as they are somewhat cosmopolite source for seeking information on agriculture and related activities. It is true because agriculture is major constituent of rural development.

From the above findings it can be seen that majority of the respondents 'always' used 'newspaper', as now a days, the availability is easy. Also, one of the reason for using printed 
media is that no one respondent was found 'illiterate'. It is also cheap and easy access to day to day happenings, government activities etc. Agricultural magazines were also used 'sometimes' by considerable respondents as it is a good source of information. Newsletters are also used by considerable number of respondent as a source of information. Television use was found more than radio due to advancements. Also, the use of media like computer, internet was negligible, due to the lack of knowledge about handling of advanced media.

'Agriculture diary' was also used 'sometimes' by some respondent due to the fact that, area under study comes nearer to 'agriculture university'.

From the findings, among the extension teaching methods, posters, charts, circulars were found effective. It is clear that majority of the respondents used 'group discussion', as any developmental programme before implementation requires to be clear among the members, as well as, villagers, so it is more used. It was followed by demonstrations, trainings, visits, campaigns, and rallies etc. which are organized by concerned government staff. Thus, the information regarding agriculture, allied sectors, development programmes, sanitation, education etc. is disseminated effectively through media. PRIs may make suitable policy to increase the use of least used media among woman PRIs members.

\section{Media utilization by woman members of PRIs}

Being the member of PRI, one has to look after different spheres of rural development. Agriculture, education, health, water supply, woman and child welfare and works are the major areas as far as the rural development of a village is concerned.
With this view, an attempt was also made in the present study to understand as to what extent available source was utilized by respondent members to obtain area wise information.

The data regarding media utilization by woman members of PRIs are given in Table 3.

It is seen from Table 3 that among the personal localite sources, family members, relatives, Grampanchayat members and local leaders were used by the woman members for seeking information. The information gained from family member's was utilized for educational and health (100.00 per cent), agriculture (98.21 per cent) and woman and child welfare ( 87.50 per cent) activities. The information gained from relatives was utilized mainly for educational activities (53.33 per cent). Further, the information obtained from Grampanchayat members was utilized for agriculture (67.30 per cent), health(84.61 per cent), women and child welfare (42.30 per cent) activities, while that received from local leaders was mainly utilized for women and child welfare (52.00 per cent), educational (48.00 per cent) and other development activities (45.00 per cent). Woman members utilized the information procured from Grampanchayat members for health (84.61 per cent), agriculture (67.30 per cent), followed by women and child welfare (42.30 per cent) activities.

In case of personal cosmopolite sources from Department of Agriculture 95.65 per cent respondents utilized information received from Agriculture Assistants, followed by Circle Agriculture Officer (97.02 per cent) and Taluka Agriculture Officer (90.62 per cent) for agriculture.

Among the personal cosmopolite sources in Panchayat Raj Institutions, 'Gramsevak' was 
the major source of information and 86.66 per cent respondents utilized the information from them for agriculture. The proportion of respondents utilizing the information provided for agriculture from Agriculture Officer was 72.34 per cent. Also, 76.40 per cent respondents utilized information from Block Development Officer for women and child welfare, followed by education (42.69 per cent).

Majority of the respondents contacted the SMSs from the Agricultural Universities and the information received from them was utilized for agriculture (60.00 per cent) and for other purpose that is, for arranging exhibitions, demonstrations and rallies (93.33 per cent).

Among the mass media, majority (94.44 per cent) gained information from newspapers which was utilized for other purposes like, political issues, schemes by private sectors and other developmental issues, followed by health (59.25 per cent) and agriculture (53.70 per cent). They also used agricultural magazines for information on agriculture (97.70 per cent). Information on radio was used for agriculture by 52.05 per cent respondents. Also, information on television was used for activities like agriculture (11.11 per cent) and health (35.18 per cent). It is revealed from the Table 3 that among various extension teaching methods, utilization of poster for information on health was made by 67.30 per cent respondents, followed by education (55.76 per cent). Information from Circulars was used mainly for agriculture (54.54 per cent) and education (52.72 per cent).

Information gained through group discussions was utilized for educational purpose by 31.48 per cent respondents, followed by agriculture (29.62 per cent). Information gained through trainings was utilized for women and child welfare (73.91 per cent), while that through exhibitions was (94.11 per cent) used for agriculture. Majority, (93.57 per cent) of the respondents utilized the information gained from campaigns for health and that from rallies for agriculture (65.26 per cent). The information gained from field visits was utilized for agriculture (100.00 per cent).

It can be said that the personal localite contacts were utilized by majority of respondents, as respondents were women. Also, maximum number of media was utilized for seeking information on important rural developmental activities like agriculture, health, education and women and child welfare. In case of personal cosmopolite contacts, these were less utilized. Also, the newspaper and television were considerably utilized for gaining information on various rural development activities.

Table.1 Distribution of the respondents according to the extent of media used by them for seeking information on rural development

\begin{tabular}{|l|l|c|c|}
\hline SI. No. & Extent of media use (score) & \multicolumn{2}{|c|}{ Respondents (N=112) } \\
\cline { 3 - 4 } & & Number & Percentage \\
\hline 1. & Low (upto 24) & 17 & 15.18 \\
\hline 2. & Medium (25 to 38) & 79 & 70.54 \\
\hline 3. & High (39 and above) & 16 & 14.28 \\
\hline & Average (31.65) Total & 112 & 100.00 \\
\hline
\end{tabular}


Table.2 Type and frequency of media use by PRI's woman members for seeking information on rural development

\begin{tabular}{|c|c|c|c|c|c|}
\hline \multirow{2}{*}{$\begin{array}{l}\text { Sl. } \\
\text { No. }\end{array}$} & \multirow[t]{2}{*}{ Media } & \multicolumn{3}{|c|}{ Extent of media use and utilization } & \multirow{2}{*}{$\begin{array}{l}\text { Total } \\
\text { Total }\end{array}$} \\
\hline & & Always & Sometimes & Never & \\
\hline 1 & 2 & 3 & 4 & 5 & 6 \\
\hline I) & \multicolumn{5}{|c|}{ Personal contact : i) Localite } \\
\hline 1. & Family member & 104(92.86) & $8(7.14)$ & - & $112(100.00)$ \\
\hline 2. & Relative & $15(13.39)$ & $90(80.36)$ & $7(6.25)$ & $112(100.00)$ \\
\hline 3. & Friends & $10(8.93)$ & 77(68.75) & $25(22.32)$ & $112(100.00)$ \\
\hline 4. & Neighbour & $36(32.14)$ & $54(48.22)$ & $22(19.64)$ & $112(100.00)$ \\
\hline 5. & Progressive farmer & $10(8.93)$ & $87(77.68)$ & $15(13.39)$ & $112(100.00)$ \\
\hline 6. & Local leaders & $50(44.64)$ & $50(44.64)$ & $12(10.72)$ & $112(100.00)$ \\
\hline 7. & G.P. members & $63(56.25)$ & $41(36.60)$ & $8(7.15)$ & $112(100.00)$ \\
\hline 8. & P.S. members & $27(24.10)$ & $12(10.72)$ & $73(65.18)$ & $112(100.00)$ \\
\hline 9. & Z.P. members & 13(11.61) & $17(15.18)$ & $82(73.21)$ & $112(100.00)$ \\
\hline 10. & P.S. President & $24(21.42)$ & $12(10.72)$ & $76(67.86)$ & $112(100.00)$ \\
\hline 11. & Z. P. Chairperson & 15(13.39) & $9(8.04)$ & $88(78.57)$ & $112(100.00)$ \\
\hline ii) & \multicolumn{5}{|c|}{ Cosmopolite : a) Department of Agriculture } \\
\hline 12. & Agriculture Assistant & $3(2.68)$ & $66(58.93)$ & 43(38.39) & 112(100.00) \\
\hline 13. & Agriculture Supervisor & - & $15(13.39)$ & $97(86.61)$ & $112(100.00)$ \\
\hline 14. & Agriculture officer & $10(8.93)$ & $91(81.25)$ & $11(9.82)$ & $112(100.00)$ \\
\hline 15. & Taluka A.O. & $12(10.71)$ & $52(46.43)$ & $48(42.86)$ & $112(100.00)$ \\
\hline 16. & Subdivisional A.O. & $2(1.78)$ & $9(8.03)$ & $101(90.19)$ & $112(100.00)$ \\
\hline 17. & District S.A.O. & $4(3.58)$ & $13(11.60)$ & $95(84.82)$ & $112(100.00)$ \\
\hline b) & \multicolumn{5}{|c|}{ Panchayat Raj staff / officers } \\
\hline 18. & Gramsevak & $74(66.07)$ & $31(27.68)$ & $7(6.25)$ & $112(100.00)$ \\
\hline 19. & Agril.Extension Officer & $14(12.50)$ & $48(42.86)$ & $50(44.64)$ & $112(100.00)$ \\
\hline 20. & Agriculture Officer & 19(16.97) & $75(66.96)$ & $18(16.07)$ & $112(100.00)$ \\
\hline 21. & B. D. O. & $21(18.75)$ & $68(60.72)$ & $23(20.53)$ & $112(100.00)$ \\
\hline 22. & Project Officer & $1(0.89)$ & $20(17.86)$ & $91(81.25)$ & $112(100.00)$ \\
\hline c) & \multicolumn{5}{|c|}{ Agricultural universities } \\
\hline 23. & Agriculture Assistant & $1(0.89)$ & $5(4.47)$ & 106(94.64) & $112(100.00)$ \\
\hline 24. & Agriculture Supervisor & - & - & $112(100.00)$ & $112(100.00)$ \\
\hline 25. & Agriculture Officer & - & - & $112(100.00)$ & $112(100.00)$ \\
\hline 26. & SMSs & $2(1.78)$ & $28(25.00)$ & $82(73.22)$ & $112(100.00)$ \\
\hline 27. & Scientists & $2(1.78)$ & $15(13.39)$ & $95(84.83)$ & $112(100.00))$ \\
\hline d) & \multicolumn{5}{|l|}{ Bank Officers } \\
\hline 28. & Agriculture Officer & - & - & $112(100.00)$ & $112(100.00)$ \\
\hline 29. & Branch Manager & - & $5(4.46)$ & 107(95.54) & $112(100.00)$ \\
\hline II) & \multicolumn{5}{|l|}{ Mass media } \\
\hline 30. & Newspaper & $83(74.10)$ & $25(22.33)$ & $4(3.57)$ & $112(100.00)$ \\
\hline
\end{tabular}




\begin{tabular}{|c|c|c|c|c|c|}
\hline 31. & Agricultural magazine & $4(3.58)$ & $83(74.10)$ & $25(22.33)$ & $112(100.00)$ \\
\hline 32. & Journals & - & $13(11.61)$ & $99(88.39)$ & $112(100.00)$ \\
\hline 33. & Newsletters & $41(36.61)$ & $65(58.04)$ & $6(5.35)$ & $112(100.00)$ \\
\hline 34. & Books on schemes & $2(1.78)$ & $29(25.89)$ & $81(72.33)$ & $112(100.00)$ \\
\hline 35. & Agriculture diary & $1(0.89)$ & $44(39.28)$ & $67(59.83)$ & $112(100.00)$ \\
\hline 36. & Radio & $28(25.00)$ & $45(40.18)$ & $39(34.82)$ & $112(100.00)$ \\
\hline 37. & Television & $67(59.82)$ & $41(36.61)$ & $4(3.57)$ & $112(100.00)$ \\
\hline 38. & Computer & $3(2.67)$ & $20(17.86)$ & $89(79.47)$ & $112(100.00)$ \\
\hline 39. & Internet & $1(0.89)$ & $13(11.61)$ & $98(87.50)$ & $112(100.00)$ \\
\hline III) & \multicolumn{5}{|c|}{ Extension teaching methods } \\
\hline 40. & Posters & $2(1.78)$ & 102(91.08) & $8(7.14)$ & $112(100.00)$ \\
\hline 41. & Circulars & $76(67.86)$ & $34(30.36)$ & $2(1.78)$ & $112(100.00)$ \\
\hline 42. & Charts & - & $68(60.72)$ & 44(39.28) & $112(100.00)$ \\
\hline 43. & Graphs & - & $7(6.25)$ & 105(93.75) & $112(100.00)$ \\
\hline 44. & Tape recorder & $2(1.78)$ & $4(3.58)$ & 106(94.64) & $112(100.00)$ \\
\hline 45. & Film & - & $3(2.69)$ & 109(97.31) & $112(100.00)$ \\
\hline 46. & Visits & $2(1.78)$ & $51(45.54)$ & $59(52.68)$ & $112(100.00)$ \\
\hline 47. & Group discussion & $68(60.71)$ & $40(35.71)$ & $4(3.58)$ & $112(100.00)$ \\
\hline 48. & Demonstration & $3(2.68)$ & $37(33.04)$ & $72(64.28)$ & $112(100.00)$ \\
\hline 49. & Trials & - & $5(4.46)$ & $107(95.54)$ & $112(100.00)$ \\
\hline 50. & Workshops & $1(0.89)$ & $4(3.57)$ & $107(95.54)$ & $112(100.00)$ \\
\hline 51. & Training & $3(2.68)$ & $89(79.46)$ & $20(17.86)$ & $112(100.00)$ \\
\hline 52. & Exhibition & - & $102(91.08)$ & $10(8.92)$ & $112(100.00)$ \\
\hline 53. & Seminar & - & $6(5.36)$ & $106(94.64)$ & $112(100.00)$ \\
\hline 54. & Campaign & $2(1.78)$ & $107(95.54)$ & $3(2.68)$ & $112(100.00)$ \\
\hline 55. & Rally & - & $95(84.82)$ & $17(15.18)$ & $112(100.00)$ \\
\hline 56. & Field visits & - & $43(38.39)$ & $69(61.61)$ & $112(100.00)$ \\
\hline
\end{tabular}

(Figures in parent heses are percentages to total number of respondents.) 
Table.3 Utilization of information gained by woman members of PRIs from different media

\begin{tabular}{|c|c|c|c|c|c|c|c|c|}
\hline \multirow[b]{2}{*}{ Sl. no. } & \multirow[b]{2}{*}{ Media } & \multicolumn{7}{|c|}{ Utilization of media } \\
\hline & & Agricultural & Educational & Health & Water Supply & $\begin{array}{l}\text { Women and } \\
\text { child welfare }\end{array}$ & Construction & Other \\
\hline 1 & 2 & 3 & 4 & 5 & 6 & 7 & 8 & 9 \\
\hline I) & \multicolumn{8}{|c|}{ Personal contact : i) Localite } \\
\hline 1. & Family member $\quad(n=112)$ & $\begin{array}{l}110 \\
(98.21)\end{array}$ & $\begin{array}{l}112 \\
(100.00)\end{array}$ & $\begin{array}{l}112 \\
(100.00)\end{array}$ & $\begin{array}{l}7 \\
(6.25)\end{array}$ & $\begin{array}{l}98 \\
(87.50)\end{array}$ & $\begin{array}{l}30 \\
(26.78)\end{array}$ & $\begin{array}{l}88 \\
(78.57)\end{array}$ \\
\hline 2. & $\begin{array}{l}\text { Relative } \\
(n=105)\end{array}$ & $\begin{array}{l}40 \\
(38.09)\end{array}$ & $\begin{array}{l}56 \\
(53.33)\end{array}$ & $\begin{array}{l}32 \\
(30.47)\end{array}$ & - & $\begin{array}{l}15 \\
(14.28)\end{array}$ & - & - \\
\hline 3. & $\begin{array}{l}\text { Friends } \\
(\mathrm{n}=87)\end{array}$ & $\begin{array}{l}20 \\
(22.98)\end{array}$ & $\begin{array}{l}64 \\
(73.56)\end{array}$ & $\begin{array}{l}60 \\
(68.96)\end{array}$ & $\begin{array}{l}2 \\
(2.29)\end{array}$ & $\begin{array}{l}40 \\
(45.97)\end{array}$ & $\begin{array}{l}4 \\
(4.59)\end{array}$ & $\begin{array}{l}26 \\
(29.88)\end{array}$ \\
\hline 4. & $\begin{array}{l}\text { Neighbors } \\
(n=90)\end{array}$ & $\begin{array}{l}45 \\
(50.00)\end{array}$ & $\begin{array}{l}58 \\
(64.44)\end{array}$ & $\begin{array}{l}70 \\
(77.77)\end{array}$ & - & - & $\begin{array}{l}2 \\
(2.22)\end{array}$ & - \\
\hline 5. & $\begin{array}{l}\text { Progressive farmer } \\
(n=97)\end{array}$ & $\begin{array}{l}97 \\
(100)\end{array}$ & $\begin{array}{l}4 \\
(4.12)\end{array}$ & $\begin{array}{l}2 \\
(2.06)\end{array}$ & $\begin{array}{l}22 \\
(22.68)\end{array}$ & - & $\begin{array}{l}12 \\
(12.37)\end{array}$ & - \\
\hline 6. & $\begin{array}{l}\text { Local leaders } \\
(\mathrm{n}=100)\end{array}$ & $\begin{array}{l}12 \\
(12.00)\end{array}$ & $\begin{array}{l}48 \\
(48.00)\end{array}$ & - & $\begin{array}{l}8 \\
(8.00)\end{array}$ & $\begin{array}{l}52 \\
(52.00)\end{array}$ & $\begin{array}{l}10 \\
(10.00)\end{array}$ & $\begin{array}{l}45 \\
(45.00)\end{array}$ \\
\hline 7. & $\begin{array}{l}\text { G.P. members } \\
(n=104)\end{array}$ & $\begin{array}{l}70 \\
(67.30)\end{array}$ & $\begin{array}{l}32 \\
(30.76)\end{array}$ & $\begin{array}{l}88 \\
(84.61)\end{array}$ & $\begin{array}{l}12 \\
(11.53)\end{array}$ & $\begin{array}{l}44 \\
(42.30)\end{array}$ & $\begin{array}{l}18 \\
(17.30)\end{array}$ & $\begin{array}{l}24 \\
(23.07)\end{array}$ \\
\hline 8. & $\begin{array}{l}\text { P.S. members } \\
(\mathrm{n}=39)\end{array}$ & $\begin{array}{l}10 \\
(25.64)\end{array}$ & $\begin{array}{l}16 \\
(41.02)\end{array}$ & $\begin{array}{l}12 \\
(30.76)\end{array}$ & $\begin{array}{l}18 \\
(46.15)\end{array}$ & $\begin{array}{l}32 \\
(82.05)\end{array}$ & $\begin{array}{l}6 \\
(15.38)\end{array}$ & - \\
\hline 9. & $\begin{array}{l}\text { Z.P. members } \\
(n=30)\end{array}$ & - & $\begin{array}{l}12 \\
(40.00)\end{array}$ & $\begin{array}{l}18 \\
(60.00)\end{array}$ & $\begin{array}{l}15 \\
(50.00)\end{array}$ & $\begin{array}{l}20 \\
(66.66)\end{array}$ & $\begin{array}{l}8 \\
(26.66)\end{array}$ & $\begin{array}{l}19 \\
(63.33)\end{array}$ \\
\hline 10. & $\begin{array}{l}\text { P.S. President } \\
(n=36)\end{array}$ & - & - & $\begin{array}{l}2 \\
(5.55)\end{array}$ & $\begin{array}{l}22 \\
(61.11)\end{array}$ & $\begin{array}{l}30 \\
(83.33)\end{array}$ & $\begin{array}{l}8 \\
(22.22)\end{array}$ & $\begin{array}{l}12 \\
(33.33)\end{array}$ \\
\hline 11. & $\begin{array}{l}\text { Z. P. Chairperson } \\
(\mathrm{n}=24)\end{array}$ & - & $\begin{array}{l}8 \\
(33.33)\end{array}$ & $\begin{array}{l}12 \\
(50.00)\end{array}$ & $\begin{array}{l}14 \\
(58.33)\end{array}$ & $\begin{array}{l}22 \\
(91.66)\end{array}$ & $\begin{array}{l}2 \\
(8.33)\end{array}$ & - \\
\hline
\end{tabular}




\begin{tabular}{|c|c|c|c|c|c|c|c|c|}
\hline 1 & 2 & 3 & 4 & 5 & 6 & 7 & 8 & 9 \\
\hline $\begin{array}{l}\text { ii) } \\
\text { a) }\end{array}$ & \multicolumn{8}{|l|}{$\begin{array}{l}\text { Cosmopolite : } \\
\text { Department of Agriculture }\end{array}$} \\
\hline 12. & Agriculture Assistant (n=69) & $\begin{array}{l}66 \\
(95.65)\end{array}$ & - & - & - & - & - & - \\
\hline 13. & Agriculture Supervisor $(\mathrm{n}=15)$ & $\begin{array}{l}14 \\
(93.33)\end{array}$ & - & - & $\begin{array}{l}2 \\
(14.28)\end{array}$ & - & - & - \\
\hline 14. & Circle Agriculture Officer $(\mathrm{n}=101)$ & $\begin{array}{l}98 \\
(97.02)\end{array}$ & - & - & $\begin{array}{l}12 \\
(11.88)\end{array}$ & - & - & - \\
\hline 15. & $\begin{array}{l}\text { Taluka A.O. } \\
(\mathrm{n}=64)\end{array}$ & $\begin{array}{l}58 \\
(90.62)\end{array}$ & - & - & - & - & - & - \\
\hline 16. & Subdivisional A.O. $(\mathrm{n}=11)$ & $\begin{array}{l}11 \\
(100)\end{array}$ & - & - & - & - & - & - \\
\hline 17. & $\begin{array}{l}\text { District S.A.O. } \\
(\mathrm{n}=17)\end{array}$ & $\begin{array}{l}12 \\
(70.58)\end{array}$ & - & - & $\begin{array}{l}10 \\
(58.82)\end{array}$ & - & - & - \\
\hline b) & \multicolumn{8}{|l|}{ Panchayat Raj staff/ officers } \\
\hline 18. & Gramsevak $(\mathrm{n}=105)$ & $\begin{array}{l}91 \\
(86.66)\end{array}$ & $\begin{array}{l}14 \\
(13.33)\end{array}$ & $\begin{array}{l}18 \\
(17.14)\end{array}$ & $\begin{array}{l}4 \\
(3.80)\end{array}$ & $\begin{array}{l}8 \\
(7.61)\end{array}$ & $\begin{array}{l}2 \\
(1.90)\end{array}$ & $\begin{array}{l}34 \\
(32.38)\end{array}$ \\
\hline 19. & Agril. Extension Officer $(\mathrm{n}=62)$ & $\begin{array}{l}54 \\
(87.09)\end{array}$ & - & - & - & - & $\begin{array}{l}8 \\
(12.90)\end{array}$ & $\begin{array}{l}12 \\
(19.35)\end{array}$ \\
\hline 20. & Agriculture Officer $(\mathrm{n}=94)$ & $\begin{array}{l}68 \\
(72.34)\end{array}$ & - & - & $\begin{array}{l}8 \\
(8.51)\end{array}$ & - & $\begin{array}{l}4 \\
(4.25)\end{array}$ & $\begin{array}{l}16 \\
(17.02)\end{array}$ \\
\hline 21. & $\begin{array}{l}\text { B. D. O. } \\
(n=89)\end{array}$ & $\begin{array}{l}14 \\
(15.73)\end{array}$ & $\begin{array}{l}38 \\
(42.69)\end{array}$ & $\begin{array}{l}26 \\
(29.21)\end{array}$ & - & $\begin{array}{l}68 \\
(76.40)\end{array}$ & - & $\begin{array}{l}10 \\
(11.23)\end{array}$ \\
\hline 22. & $\begin{array}{l}\text { Project Officer } \\
(n=21)\end{array}$ & - & - & - & $\begin{array}{l}2 \\
(4.76)\end{array}$ & $\begin{array}{l}16 \\
(76.19)\end{array}$ & - & - \\
\hline c) & \multicolumn{8}{|l|}{ Agricultural Universities } \\
\hline 23. & Agriculture Assistant $(\mathrm{n}=6)$ & $\begin{array}{l}4 \\
(66.66)\end{array}$ & - & - & - & - & - & $\begin{array}{l}2 \\
(33.33)\end{array}$ \\
\hline 24. & Agriculture Supervisor $(\mathrm{n}=0)$ & - & - & - & - & - & - & - \\
\hline 25. & Agriculture Officer $(\mathrm{n}=0)$ & - & - & - & - & - & - & - \\
\hline
\end{tabular}




\section{Int.J.Curr.Microbiol.App.Sci (2020) 9(6): 3221-3234}

\begin{tabular}{|c|c|c|c|c|c|c|c|c|}
\hline 1 & 2 & 3 & 4 & 5 & 6 & 7 & 8 & 9 \\
\hline 26. & $\begin{array}{l}\text { SMSs } \\
(\mathrm{n}=30)\end{array}$ & $\begin{array}{l}18 \\
(60.00)\end{array}$ & - & - & - & - & - & $\begin{array}{l}28 \\
(93.33)\end{array}$ \\
\hline 27. & $\begin{array}{l}\text { Scientists } \\
(\mathrm{n}=17)\end{array}$ & $\begin{array}{l}12 \\
(70.58)\end{array}$ & - & - & - & - & - & $\begin{array}{l}8 \\
(47.05)\end{array}$ \\
\hline d) & \multicolumn{8}{|l|}{ Bank Officers } \\
\hline 28. & Agriculture Officer $(n=0)$ & - & - & - & - & - & - & - \\
\hline 29. & $\begin{array}{l}\text { Branch Manager } \\
(\mathrm{n}=5)\end{array}$ & - & - & - & - & - & - & $\begin{array}{l}5 \\
(100.00)\end{array}$ \\
\hline II) & \multicolumn{8}{|l|}{ Mass media } \\
\hline 30. & $\begin{array}{l}\text { Newspaper } \\
(n=108)\end{array}$ & $\begin{array}{l}58 \\
(53.70)\end{array}$ & $\begin{array}{l}28 \\
(25.92)\end{array}$ & $\begin{array}{l}64 \\
(59.25)\end{array}$ & $\begin{array}{l}4 \\
(3.70)\end{array}$ & $\begin{array}{l}12 \\
(11.11)\end{array}$ & - & $\begin{array}{l}102 \\
(94.44)\end{array}$ \\
\hline 31. & $\begin{array}{l}\text { Agricultural magazine } \\
(\mathrm{n}=87)\end{array}$ & $\begin{array}{l}85 \\
(97.70)\end{array}$ & - & - & - & - & - & $\begin{array}{l}8 \\
(9.19)\end{array}$ \\
\hline 32. & $\begin{array}{l}\text { Journals } \\
(n=13)\end{array}$ & - & $\begin{array}{l}4 \\
(30.76)\end{array}$ & - & - & $\begin{array}{l}6 \\
(46.15)\end{array}$ & - & $\begin{array}{l}8 \\
(61.53)\end{array}$ \\
\hline 33. & $\begin{array}{l}\text { Newsletters } \\
(n=106)\end{array}$ & $\begin{array}{l}13 \\
(12.32)\end{array}$ & $\begin{array}{l}81 \\
(76.41)\end{array}$ & $\begin{array}{l}4 \\
(3.77)\end{array}$ & - & - & $\begin{array}{l}8 \\
(7.54)\end{array}$ & - \\
\hline 34. & Books on schemes $(n=31)$ & $\begin{array}{l}18 \\
(58.06)\end{array}$ & - & - & $\begin{array}{l}12 \\
(38.70)\end{array}$ & $\begin{array}{l}28 \\
(90.32)\end{array}$ & - & - \\
\hline 35. & Agriculture Diary $(n=45)$ & $\begin{array}{l}45 \\
(100.00)\end{array}$ & - & - & - & - & - & - \\
\hline 36. & Radio $(n=73)$ & $\begin{array}{l}38 \\
(52.05)\end{array}$ & $\begin{array}{l}20 \\
(27.39)\end{array}$ & $\begin{array}{l}32 \\
(43.83)\end{array}$ & - & $\begin{array}{l}12 \\
(16.43)\end{array}$ & - & $\begin{array}{l}20 \\
(27.39)\end{array}$ \\
\hline 37. & Television $(\mathrm{n}=108)$ & $\begin{array}{l}12 \\
(11.11)\end{array}$ & $\begin{array}{l}21 \\
(19.44)\end{array}$ & $\begin{array}{l}38 \\
(35.18)\end{array}$ & - & - & - & $\begin{array}{l}89 \\
(82.40)\end{array}$ \\
\hline 38. & Computer (CDs) $(\mathrm{n}=23)$ & - & $\begin{array}{l}23 \\
(100)\end{array}$ & - & - & - & - & - \\
\hline 39. & Internet $(n=14)$ & - & $\begin{array}{l}14 \\
(100)\end{array}$ & - & - & - & - & $\begin{array}{l}4 \\
(28.57)\end{array}$ \\
\hline
\end{tabular}




\begin{tabular}{|c|c|c|c|c|c|c|c|c|}
\hline 1 & 2 & 3 & 4 & 5 & 6 & 7 & 8 & 9 \\
\hline III) & \multicolumn{8}{|c|}{ Extension teaching methods } \\
\hline 40. & Posters $(n=104)$ & $\begin{array}{l}34 \\
(32.69)\end{array}$ & $\begin{array}{l}58 \\
(55.76)\end{array}$ & $\begin{array}{l}70 \\
(67.30)\end{array}$ & - & $\begin{array}{l}14 \\
(13.46)\end{array}$ & - & $\begin{array}{l}18 \\
(17.30)\end{array}$ \\
\hline 41. & Circulars $(\mathrm{n}=110)$ & $\begin{array}{l}60 \\
(54.54)\end{array}$ & $\begin{array}{l}58 \\
(52.72)\end{array}$ & $\begin{array}{l}21 \\
(19.09)\end{array}$ & - & $\begin{array}{l}34 \\
(30.90)\end{array}$ & $\begin{array}{l}18 \\
(16.36)\end{array}$ & $\begin{array}{l}4 \\
(3.63)\end{array}$ \\
\hline 42. & Charts $(n=68)$ & $\begin{array}{l}4 \\
(5.88)\end{array}$ & $\begin{array}{l}45 \\
(66.17)\end{array}$ & $\begin{array}{l}48 \\
(70.58)\end{array}$ & - & - & - & - \\
\hline 43. & Graphs $(n=7)$ & - & $\begin{array}{l}4 \\
(57.14)\end{array}$ & - & - & - & - & $\begin{array}{l}5 \\
(71.42)\end{array}$ \\
\hline 44. & $\begin{array}{l}\text { Tape recorder }(n=6) \\
\text { (Audio Cassettes) }\end{array}$ & $\begin{array}{l}2 \\
(33.33)\end{array}$ & $\begin{array}{l}4 \\
(66.66)\end{array}$ & - & - & - & - & - \\
\hline 45. & Film $(n=3)$ & - & - & $\begin{array}{l}3 \\
(100)\end{array}$ & - & $\begin{array}{l}2 \\
(66.66)\end{array}$ & - & - \\
\hline 46. & Visits $(n=53)$ & $\begin{array}{l}48 \\
(90.56)\end{array}$ & $\begin{array}{l}12 \\
(22.64)\end{array}$ & - & - & $\begin{array}{l}4 \\
(7.54)\end{array}$ & - & - \\
\hline 47. & Group discussion $(\mathrm{n}=108)$ & $\begin{array}{l}32 \\
(29.62)\end{array}$ & $\begin{array}{l}34 \\
(31.48)\end{array}$ & $\begin{array}{l}18 \\
(16.66)\end{array}$ & $\begin{array}{l}12 \\
(11.11)\end{array}$ & $\begin{array}{l}8 \\
(7.40)\end{array}$ & $\begin{array}{l}4 \\
(3.70)\end{array}$ & - \\
\hline 48. & Demonstration $(\mathrm{n}=40)$ & $\begin{array}{l}38 \\
(95.00)\end{array}$ & - & - & - & - & - & $\begin{array}{l}4 \\
(10.00)\end{array}$ \\
\hline 49. & Trials $(n=5)$ & $\begin{array}{l}4 \\
(80.00)\end{array}$ & - & - & - & - & - & $\begin{array}{l}2 \\
(40.00)\end{array}$ \\
\hline 50. & Workshops $(n=5)$ & - & $\begin{array}{l}4 \\
(80.00)\end{array}$ & - & - & $\begin{array}{l}3 \\
(60.00)\end{array}$ & - & - \\
\hline 51. & Training $(\mathrm{n}=92)$ & $\begin{array}{l}12 \\
(13.04)\end{array}$ & $\begin{array}{l}14 \\
(15.21)\end{array}$ & - & - & $\begin{array}{l}68 \\
(73.91)\end{array}$ & - & - \\
\hline 52. & Exhibition $(\mathrm{n}=102)$ & $\begin{array}{l}96 \\
(94.11)\end{array}$ & - & - & - & - & - & $\begin{array}{l}12 \\
(11.76)\end{array}$ \\
\hline 53. & Seminar $(n=6)$ & - & - & $\begin{array}{l}4 \\
(6.66)\end{array}$ & - & $\begin{array}{l}2 \\
(33.33)\end{array}$ & - & - \\
\hline 54. & Campaign $(\mathrm{n}=109)$ & - & $\begin{array}{l}20 \\
(18.34)\end{array}$ & $\begin{array}{l}102 \\
(93.57)\end{array}$ & - & $\begin{array}{l}2 \\
(1.83)\end{array}$ & - & - \\
\hline 55. & Rally (n=95) & $\begin{array}{l}62 \\
(65.26)\end{array}$ & $\begin{array}{l}12 \\
(12.63)\end{array}$ & $\begin{array}{l}14 \\
(14.73)\end{array}$ & - & $\begin{array}{l}4 \\
(4.21)\end{array}$ & - & $\begin{array}{l}18 \\
(18.94)\end{array}$ \\
\hline 56. & Field visits $(n=43)$ & $\begin{array}{l}43 \\
(100.00)\end{array}$ & - & - & $\begin{array}{l}4 \\
(9.30)\end{array}$ & - & - & - \\
\hline
\end{tabular}


Extent of media use by PRI woman members for seeking information on rural development

\section{Extent of media use}

Majority (70.54 per cent) of the respondents had 'medium' extent of media use, followed by 15.18 per cent had 'low' and 14.28 per cent had 'high' extent of media use. The average score of media use was 31.65 indicating 'medium' use.

\section{Type and frequency of media use}

At overall level, it was observed that localite personal contact media were more preferred by the respondents as a large number $(92.86$ per cent) of respondents 'always' used family member for seeking information on rural development, followed by other important sources as Grampanchayat members (56.25 per cent), local leader (44.64 per cent), neighbour (32.14per cent) and Panchayat Samiti members (24.10 per cent). Regarding personal cosmopolite contact with the personnel from Department of Agriculture, it was seen that very few (10.71 per cent) respondents 'always' used Taluka Agriculture Officer for seeking information, followed by Agriculture Officer (8.93 per cent), Divisional Agriculture Officer (3.58 per cent), Agriculture Assistant (2.68 per cent). Respondents 'sometimes' used the media like Agriculture Officer (81.25 per cent), followed by Agriculture Assistant (58.93 per cent), and Taluka Agriculture officer (46.43 per cent).

Among Panchayat Raj staff/ officers, majority (66.07 per cent) of the respondents 'always' contacted 'gramsevak' for information seeking, followed by Agriculture Officers (16.97 per cent) and Block Development Officer (18.75 per cent). Majority of them 'sometimes' used the media namely as Agriculture Assistant (79.47 per cent),
Agriculture Officers (66.96 per cent) and Block Development Officer (66.72 per cent). Respondent's contact with personnel from agriculture universities for seeking information was less.

In case of mass media, majority ( 74.10 per cent) of the respondents 'always' used newspaper, followed by television (59.82 per cent) and newsletters (36.61 per cent). Majority of the respondents 'sometimes' used agriculture magazines (74.10 per cent), followed by newsletters, radio, Agricultural Diary, and television. Among extension teaching methods, majority of the respondents used circulars (67.86 per cent), followed by 60.71 per cent respondents 'always' used group discussion for information seeking. Majority (95.54 per cent) respondents 'sometimes' used campaign, followed by exhibition and posters (91.08 each), rally (84.82 per cent), training (79.46 per cent) and field visits(45.54 per cent).

\section{Media utilization by PRI woman members}

The woman PRI members used media under study for seeking information on different activities. Among those media, they used personal localite sources more than the cosmopolite sources. Family member was the major source for the information on education and health (100.00 per cent each), followed by agriculture (98.21 per cent) and women and child welfare (87.50 per cent). Respondents utilized the information on education (53.33 per cent) from relatives, on education (73.56 per cent) from friends, on health (77.77 per cent) from neighbours, on women and child welfare (52.00 per cent) from local leaders. For information on health, 84.61 per cent respondents utilized Grampanchayat members. Also, 67.30 per cent of respondents used for seeking information on agriculture. 
In case of personal cosmopolite sources from Department of Agriculture 95.65 per cent respondents utilized information received from Agriculture Assistants for agriculture, followed by Circle Agriculture Officer (97.02 per cent) and Taluka Agriculture Officer (90.62 per cent).

Among the personal cosmopolite sources in Panchayat Raj Institutions, Gramsevak was the major source of information and 86.66 per cent respondents utilized the information on agriculture from them.

Among the mass media, majority (94.44 per cent) gained information from newspapers which was utilized for other purposes like, political issues, schemes by private sectors and other developmental issues, followed by health (59.25 per cent) and agriculture (53.70 per cent). They also used agricultural magazines for information on agriculture (97.70 per cent).

It was revealed that among various extension teaching methods, utilization of poster for information on health was made by 67.30 per cent respondents, followed by education (55.76 per cent). Information from circulars was used mainly for agriculture (54.54 per cent) and education (52.72 per cent).

\section{Implications}

The suggestions reported by respondents give an insight into the areas where improvement is needed, so as to improve their extent of media use and utilization will help in increasing their knowledge about rural development. The concerned agencies may adopt suitable measures to improve this situation.

\section{References}

Chandrashekhar, B. K. and Inbanthan, Anand (1991). 'Profile and participation of Zilla Parishad and Mandal Panchayat Members' A case of Karnatka. Journal of Rural Development. 10(5): 575-589.

Jhamtani, Anita., Singh, Premalata., Sharma, Nishi., and Singh B. (2001). 'Women in panchayats-perceptual roles'. Indian Journal of Extension Education 37 : 12.

Kubde, V.R., Kalantri, L.B. and Sarode, L.V. (1990). 'Background profile of members of Grampanchayats and their opinion about the institution'. Maharashtra Journal of Extension Education. 11: 252-255.

Mulla, Shabana, H. (2006). 'A study of role perception and performance of the women members of grampanchayat from Hatkanagle taluka of Kolhapur district.' M.Sc (Agri) Thesis, Mahatma Phule Krishi Vidyapeeth, Rahuri (MS).

Pujari, Varsha. (2006). 'A study on training needs of Panchayat women members in Ratnagiri district,' M. Sc. (Agri.) Thesis, Dr. Balasaheb Sawant Konkan Krishi Vidyapeeth, Dapoli, Dist. Ratnagiri (MS).

\section{How to cite this article:}

Kanase Pallavi, S., R.B. Kalamkar, S.P. Gaikwad and Chorge, K.V. 2020. Extent of Media Use by Women Members of PRIs for Seeking Information on Rural Development. Int.J.Curr.Microbiol.App.Sci. 9(06): 3221-3234. doi: https://doi.org/10.20546/ijcmas.2020.906.385 